\section{Genetic and pharmacological disruption of the TEAD-YAP complex suppresses the oncogenic activity of YAP}

\author{
Yi Liu-Chittenden, ${ }^{1,2,5}$ Bo Huang, 1,2,5 \\ Joong Sup Shim, ${ }^{3}$ Qian Chen, ${ }^{1,2}$ Se-Jin Lee, ${ }^{2}$ \\ Robert A. Anders, ${ }^{4}$ Jun O. Liu, ${ }^{3}$ and Duojia Pan ${ }^{1,2,6}$ \\ ${ }^{1}$ Howard Hughes Medical Institute, ${ }^{2}$ Department of Molecular \\ Biology and Genetics, ${ }^{3}$ Department of Pharmacology and \\ Molecular Sciences, ${ }^{4}$ Department of Pathology, Johns Hopkins \\ University School of Medicine, Baltimore, Maryland 21205, \\ USA
}

\begin{abstract}
The Drosophila TEAD ortholog Scalloped is required for Yki-mediated overgrowth but is largely dispensable for normal tissue growth, suggesting that its mammalian counterpart may be exploited for selective inhibition of oncogenic growth driven by YAP hyperactivation. Here we test this hypothesis genetically and pharmacologically. We show that a dominant-negative TEAD molecule does not perturb normal liver growth but potently suppresses hepatomegaly/tumorigenesis resulting from YAP overexpression or Neurofibromin 2 (NF2)/Merlin inactivation. We further identify verteporfin as a small molecule that inhibits TEAD-YAP association and YAPinduced liver overgrowth. These findings provide proof of principle that inhibiting TEAD-YAP interactions is a pharmacologically viable strategy against the YAP oncoprotein.
\end{abstract}

Supplemental material is available for this article.

Received March 26, 2012; revised version accepted May 11, 2012.

The Hippo tumor suppressor pathway restricts organ size in Drosophila and mammals by antagonizing the oncoprotein Yki/YAP (Zeng and Hong 2008; Pan 2010; Zhao et al. 2010; Halder and Johnson 2011). Central to the Hippo pathway is a kinase cascade leading from the protein kinase $\mathrm{Hpo} / \mathrm{Mst}$ to Yki/YAP. The Hippo kinase cascade, in turn, is regulated by a complex network of proteins, which most notably includes the Neurofibromin 2 (NF2)/Merlin tumor suppressor. Consistent with the critical role of Hippo signaling in normal tissue homeostasis, the YAP oncoprotein is overexpressed or hyperactivated in a wide spectrum of human cancers due to YAP locus amplification or genetic/epigenetic inactivation of upstream tumor suppressors. Small molecule inhibitors of YAP will not only provide important tools for

\footnotetext{
[Keywords: Hippo signaling; YAP; chemical biology; oncogene]

${ }^{5}$ These authors contributed equally to this work.

${ }^{6}$ Corresponding author

E-mail djpan@jhmi.edu

Article published online ahead of print. Article and publication date are online at http://www.genesdev.org/cgi/doi/10.1101/gad.192856.112.
}

pharmacological manipulation of Hippo signaling, but also bear tremendous potential for developing therapeutic drugs against human cancers caused by defective Hippo signaling.

As a transcriptional coactivator, YAP has been reported to bind to several DNA-binding transcription factors (for review, see Pan 2010). Among the reported YAP partners, the TEAD/TEF transcription factors are best characterized (Vassilev et al. 2001; Chen et al. 2010; Li et al. 2010). Genetic studies in Drosophila revealed an interesting property of its single TEAD ortholog, Scalloped (Sd): While $\mathrm{Sd}$ is required for tissue overgrowth driven by hyperactivated Yki, Sd (but not Yki) is largely dispensable for normal tissue growth (Huang et al. 2005; Wu et al. 2008). Thus, Sd/TEAD may belong to a growing list of genes that contribute to "non-oncogene addiction"genes that are not mutated in cancers but are critically required for cancer growth (Luo et al. 2009). The dispensability of Sd for normal growth in Drosophila suggests that the mammalian TEAD factors may be ideal targets for selective inhibition of oncogenic growth driven by YAP hyperactivation with minimal effects on normal tissue homeostasis.

Although previous studies have shown that the TEAD factors are required for YAP's oncogenic activity in cell cultures (Zhao et al. 2008), whether the TEAD factors (or any of the other reported YAP partners) are required for YAP-mediated tumorigenesis has not been determined in intact mammalian tissues. It also remains to be seen whether inhibition of the mammalian TEAD factors, like loss of Drosophila Sd, has minimal impact on normal tissue homeostasis and physiology. Such information will shed light on the "therapeutic window" of pharmacological strategies aimed at disrupting the TEAD-YAP complex as a selective means against YAP-driven tumorigenesis. Using a combination of genetic suppression in transgenic mice and discovery of lead compounds with in vitro and in vivo activities, we provide here proof of principle that inhibiting TEAD-YAP interactions is a promising and pharmacologically viable strategy against the YAP oncoprotein.

\section{Results and Discussion}

To test the feasibility of targeting the TEAD factors as a selective means of inhibiting YAP's oncogenic activity, we first used genetic approaches to inactivate the TEAD proteins in the mouse liver, an organ that is exquisitely sensitive to Hippo signaling (Camargo et al. 2007; Dong et al. 2007; Zhou et al. 2009; Lee et al. 2010; Lu et al. 2010; Song et al. 2010; Zhang et al. 2010). The mouse genome contains four highly homologous TEAD family members, all of which are expressed in the liver (http:// www.genenetwork.org). To overcome potential functional redundancy among family members, we attempted to block the activity of all TEAD factors by engineering a truncated form of TEAD2 that lacks its DNA-binding domain (Supplemental Fig. S1). Of note, a similar construct of Sd functions as a dominant-negative form in Drosophila (Chow et al. 2004). The dominant-negative activity of the resulting TEAD2-DN construct was confirmed in cell-based assays in which TEAD2-DN greatly suppressed YAP-mediated transactivation of Gal4-TEAD2 or Gal4TEAD4 (Supplemental Fig. S1B) as well as YAP's 
ability to promote anchorage-independent growth of HPNE (human pancreatic Nestin-expressing) cells in soft agar (Supplemental Fig. S1C,D; Dong et al. 2007). Notably, TEAD2-DN's suppressive activity is specific to YAP, as overexpression of TEAD2-DN in HPNE cells did not suppress cell proliferation or anchorage-independent growth induced by activated KRAS (Supplemental Fig. S2).

To test the activity of TEAD2-DN in vivo, we generated transgenic mice expressing TEAD2-DN under the control of a tetracycline-responsive element (TRE). We previously reported a transgenic mouse model, ApoErtTA/TRE-YAP, in which human YAP protein can be overexpressed in a liver-specific (via ApoE-rtTA) and doxycycline (Dox)-dependent (via TRE-YAP) manner (Dong et al. 2007). Crossing TRE-TEAD2-DN with ApoE-rtTA/ TRE-YAP generated transgenic mice in which YAP and TEAD2-DN were co-overexpressed in a liver-specific and Dox-dependent manner. For simplicity, these mice will be referred to as YAP/TEAD2-DN.

We showed previously that induction of YAP overexpression starting at $3 \mathrm{wk}$ of age leads to a robust and uniform expansion of liver size, whereas induction of YAP overexpression starting at birth leads to widespread development of hepatocellular carcinoma (HCC) (Dong et al. 2007). We used these experimental regimes to test the efficacy of TEAD2-DN in blocking YAP-induced overgrowth and tumorigenesis. In mice subjected to Dox treatment starting at $3 \mathrm{wk}$ of age, expression of TEAD2DN potently suppressed YAP-induced liver overgrowth (Fig. 1A,B,E), whereas in mice subjected to Dox treatment starting at birth, expression of TEAD2-DN completely abolished YAP-induced HCC formation (Fig. 1C-E). Expression of TEAD2-DN also greatly improved animal survival; in contrast to YAP transgenic mice, which had a mean survival of 7 wk after Dox treatment starting at 3 wk of age, YAP/TEAD2-DN mice showed $100 \%$ survival during a 15-wk treatment (Fig. 1F). Unlike YAPoverexpressing livers, in which hepatocytes are smaller and more densely packed than wild type, YAP/TEAD2DN livers contained hepatocytes of normal size (Fig. 1B), suggesting that TEAD2-DN may suppress YAP's ability to promote cell proliferation and/or survival. Indeed, YAP/ TEAD2-DN livers showed greatly reduced BrdU incorporation compared with YAP livers (Supplemental Fig. S3A,D). Furthermore, unlike YAP-overexpressing livers, which were resistant to Jo-2 (a Fas agonist)-induced hepatocellular apoptosis (Dong et al. 2007), Jo-2 treatment induced similar levels of apoptosis in YAP/TEAD2-DN and wild-type livers, as revealed by the widespread hemorrhage and apoptotic nuclei in liver histology and caspase 3 cleavage (Supplemental Fig. S3B,C). Thus, TEAD2-DN suppressed YAP's proproliferative and anti-apoptotic activities. At mRNA levels, TEAD2-DN reversed the upregulation of genes that have previously been reported to be transcriptionally induced in YAP transgenic livers, including Afp, Birc5/survivin, c-Myc, Sox4, Ctgf, Opn, Epcam, and $G p c 3$ (Supplemental Fig. S3E; Dong et al. 2007). Thus, TEAD2-DN can restore YAP-induced gene transcription to wild-type levels.

A trivial explanation for the observed suppression of YAP-induced phenotypes by TEAD2-DN is that TEAD2DN may somehow reduce the expression of the YAP transgene in the double-transgenic livers. We attempted to exclude this possibility by comparing YAP protein

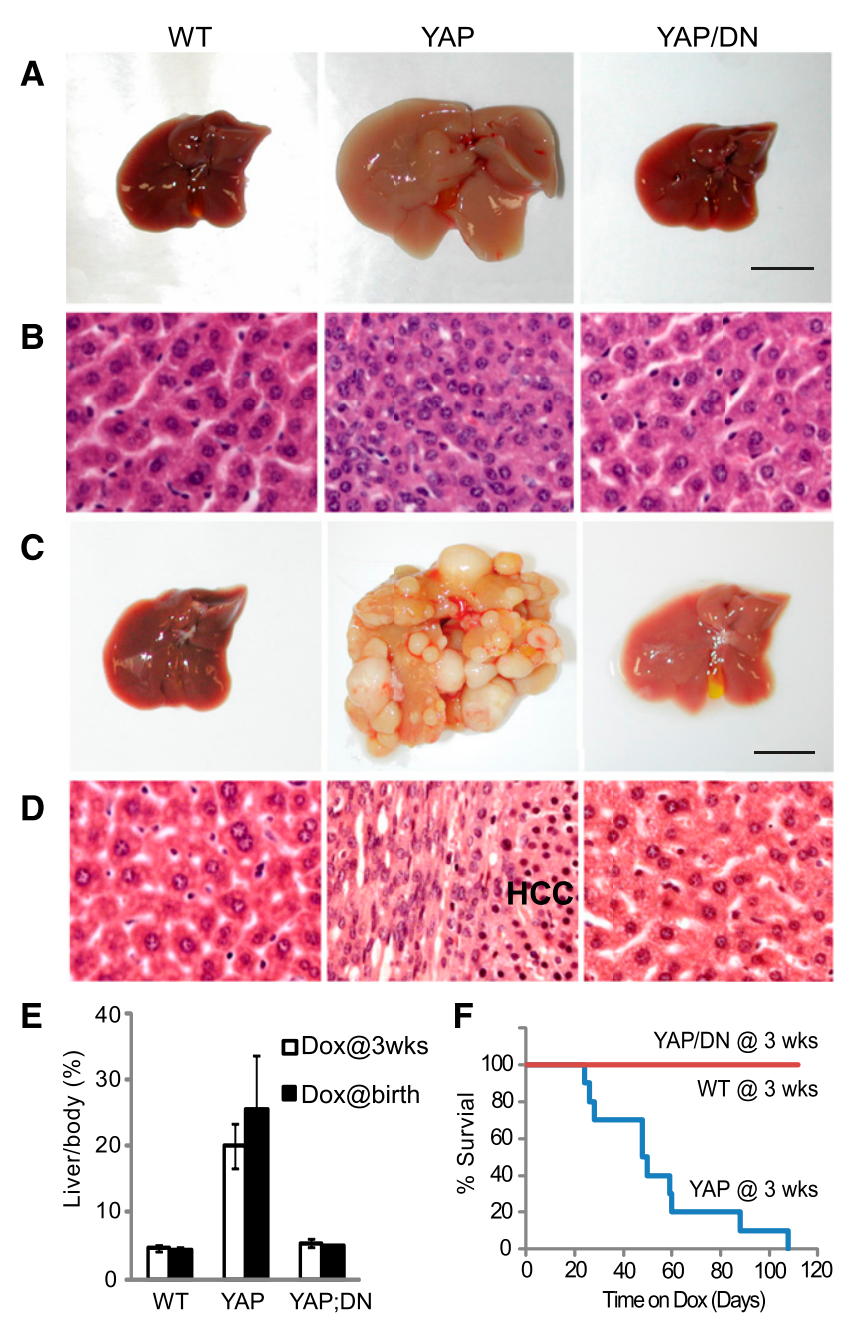

Figure 1. TEAD2-DN suppressed hepatomegaly and tumorigenesis driven by YAP overexpression. $(A, B)$ Whole amount $(A)$ and hematoxylin/eosin (H\&E) staining $(B)$ of livers from wild-type (WT), YAP, and YAP/TEAD2-DN mice treated with $0.2 \mathrm{~g} / \mathrm{L}$ Dox for $2 \mathrm{wk}$ starting at $3 \mathrm{wk}$ of age. Bar, $1 \mathrm{~cm}$. $(C, D)$ similar to $A$ and $B$ except that mice were treated with $1 \mathrm{~g} / \mathrm{L}$ Dox for $8 \mathrm{wk}$ starting at birth. $(E)$ Quantification of liver-to-body weight ratio for animals analyzed in $A$ and $C$. Values are mean \pm SEM; $n \geq 3$ for each data point. $(F)$ Survival curves of wild-type, YAP, and YAP/TEAD2-DN mice subjected to $0.2 \mathrm{~g} / \mathrm{L}$ Dox treatment starting at $3 \mathrm{wk}$ of age.

levels in YAP versus YAP/TEAD2-DN livers. On the contrary, however, we found that YAP/TEAD2-DN livers always showed significantly increased levels of YAP proteins compared with YAP transgenic livers, irrespective of the length of Dox treatment (Supplemental Fig. S4A). Quantitative real-time PCR analysis revealed similar levels of human YAP mRNA (from the YAP transgene) and endogenous mouse Yap mRNA in both genotypes (Supplemental Fig. S4B), suggesting that TEAD2-DN may increase YAP protein levels through a post-transcriptional mechanism; for example, by binding and stabilizing YAP. We tested this hypothesis in HEK293 cells. Indeed, when a GFP-tagged YAP was coexpressed with an increasing amount of HA-tagged TEAD2 or TEAD2-DN, both the exogenous GFP-YAP and the endogenous YAP protein levels were increased (Supplemental Fig. S4C). We also compared the half-life of wild-type YAP and YAP ${ }^{\mathrm{S} 94 \mathrm{~A}}$, 
a mutant form of YAP that is defective in TEAD binding (Zhao et al. 2008). YAP ${ }^{\text {S94A }}$ had a shorter half-life than wild-type YAP (Supplemental Fig. S4D), further supporting a role for TEAD in binding and stabilizing YAP.

Next, we tested TEAD2-DN in NF2/Merlin-deficient livers, wherein liver overgrowth and tumorigenesis is driven by activation of endogenous YAP, rather than overexpression of exogenous YAP (Zhang et al. 2010). For this purpose, we generated $A l b-C r e ; N f 2^{f_{l o x} / f l o x 2} ; A l b$ rtTA; TEAD2-DN mice (abbreviated as Nf2 TEAD2-DN for simplicity) in which TEAD2-DN can be expressed in a Dox-dependent manner in Nf2 mutant livers. Two Dox treatment regimes were used: a 7-wk treatment starting at gestation, and a 1-yr treatment starting at birth. Expression of TEAD2-DN significantly suppressed the overgrowth of $N f 2$ mutant livers in both conditions (Fig. $2 \mathrm{~A}, \mathrm{~B})$. In the 7-wk treatment group, TEAD2-DN abolished the development of bile duct hamartoma that were otherwise detectable (with $100 \%$ penetrance) on the surface of $N f 2$ mutant liver lobes (Fig. 2A). In the 1-yr treatment group, Nf2-deficient livers typically showed expansive bile duct hamartoma comprised of masses of cytokeratin (CK)-positive biliary epithelial cells (BECs) that have invaded deep into the liver parenchyma, as well as HCC (with $100 \%$ penetrance) and greatly increased liver weight (Fig. 2B-E). In 1-yr-old Nf2 TEAD2-DN livers, hamartoma development was greatly suppressed and no HCC was observed (Fig. 2B-D), resulting in a liver size comparable with that of wild-type mice (Fig. 2E). Consistent with phenotypic suppression, TEAD2-DN potently suppressed the up-regulation of YAP target genes in Nf2-deficient livers (Supplemental Fig. S5) in much the same way that TEAD2-DN reversed the up-regulation of these genes in YAP-overexpressing livers (Supplemental Fig. S3E). Importantly, overexpression of TEAD2-DN by itself (using Alb-rtTA; TRE-TEAD2-DN mice) did not affect liver size, liver histology, or expression of YAP target genes (Supplemental Fig. S6). Thus, much like in Drosophila, where $\mathrm{Sd}$ is required for overgrowth driven by hyperactive Yki but is dispensable for normal growth in most imaginal tissues, TEAD2-DN can prevent hepatomegaly and liver tumorigenesis caused by YAP hyperactivation without compromising normal liver homeostasis.

The striking ability of TEAD2-DN to suppress hepatomegaly and liver tumorigenesis caused by YAP overexpression or loss of NF2, combined with the inconsequentiality of TEAD2-DN on normal liver homeostasis, suggests that the TEAD-YAP transcription factor complex may offer an ideal drug target for selective inhibition of YAP-induced tumorigenesis. Thus, small molecules that inhibit the physical association between YAP and TEAD may provide a pharmacological approach for inhibiting TEAD function. To screen for such inhibitors, we took advantage of an established luciferase reporter assay in which transcriptional activity of a Gal4-TEAD4 fusion is stimulated by YAP (Zhao et al. 2008). Using this reporter assay, we screened the Johns Hopkins Drug Library, a collection of $>3300$ drugs that have been approved by the United States Food and Drug Administration or its foreign counterparts or have entered phase II clinical trials (Chong et al. 2007). We identified 71 hits that inhibited luciferase activity by $>30 \%$ at a concentration of $10 \mu \mathrm{M}$ (Fig. 3A). Three compounds (protoporphyrin IX [PPIX], hematoporphyrin [HP], and verteporfin [VP]) stood out among the top hits, as they all belong to the porphyrin family, which are

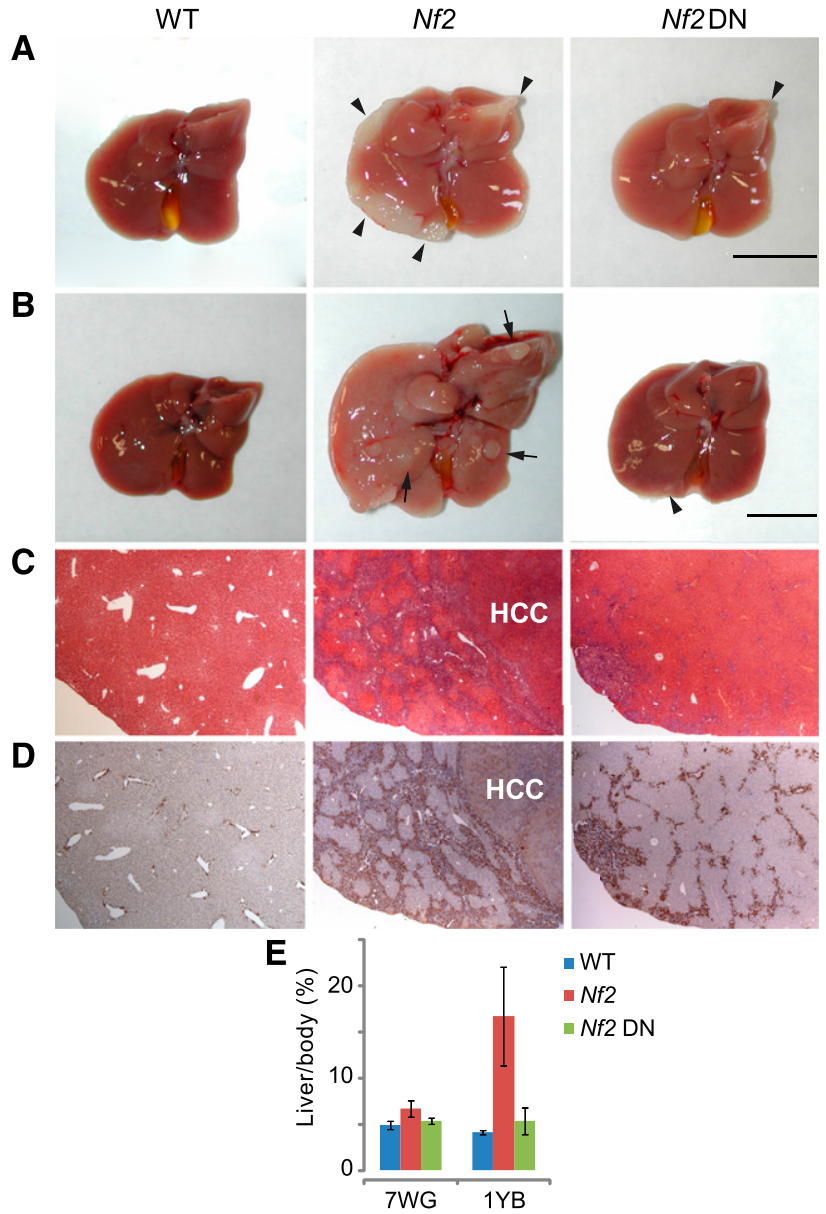

Figure 2. TEAD2-DN suppressed Nf2-deficient phenotypes in the liver. $(A)$ Livers from wild-type, Nf2, and Nf2 TEAD2-DN mice subjected to $2 \mathrm{~g} / \mathrm{L}$ Dox treatment for $7 \mathrm{wk}$ starting at gestation. Note the presence of thick hamartomas (arrowheads) at the edge of the Nf2 mutant liver. Only a remnant hamartoma (arrowhead) was visible in the Nf2 TEAD2-DN liver. Bar, $1 \mathrm{~cm}$. (B) Similar to $A$ except that mice were subjected to Dox treatment for 1 yr starting at birth. Note the massive overgrowth and the presence of multiple HCC (arrows) in the Nf2 mutant liver and the near-normal appearance of the Nf2 TEAD2-DN liver. Note that bile duct hamartomas, which appeared first at the edge of the Nf2 mutant liver (shown in A), have invaded deep into the liver parenchyma by 1 yr of age, giving the $N f 2$ mutant liver an overall pale color. Only a remnant hamartoma (arrowhead) was visible in the Nf2 TEAD2-DN liver. $(C, D) \mathrm{H} \& \mathrm{E}(C)$ and $C K(D)$ staining of liver sections from $B$. Note the presence of deep-penetrating CK-positive bile duct hamartoma and CK-negative HCC in the Nf2 mutant liver. Also note the greatly reduced bile duct hyperplasia and the absence of HCC in the Nf2 TEAD2-DN liver. $(E)$ Quantification of liver-to-body weight ratio for animals analyzed in $A$ and $B$. (7WG) Dox treatment for 7 wk starting at gestation; (1YB) Dox treatment for 1 yr starting at birth. $n \geq 6$ for each data point. In both conditions, the overgrowth of $N f 2$ mutant livers was significantly suppressed in Nf2 TEAD2-DN livers $(P=1 \times$ $10^{-5}$ and $1 \times 10^{-7}$, respectively).

aromatic heterocyclic cyclic molecules composed of four modified pyrrole units interconnected at their $\alpha$ carbon atoms via methine bridges. Coincidently, the same three porphyrin molecules were also identified as top hits in an independent FRET-based Yki-Sd interaction screen (data not shown). To confirm that these hits indeed disrupted physical interactions between YAP and TEAD, we per- 
A
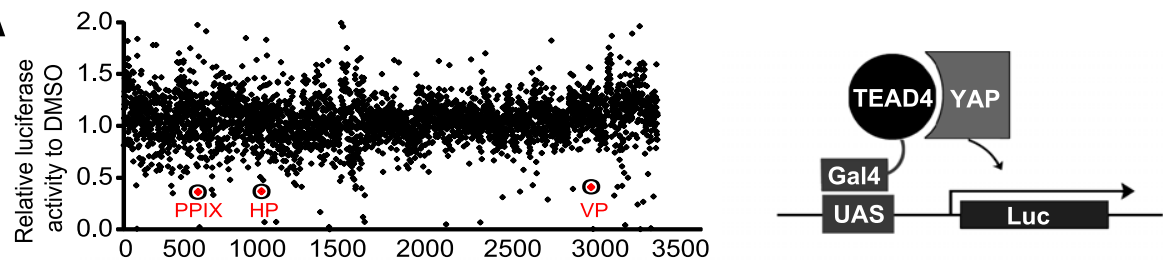

B
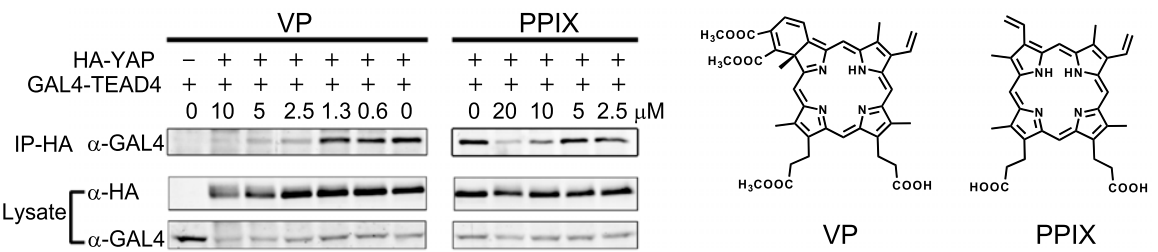

C
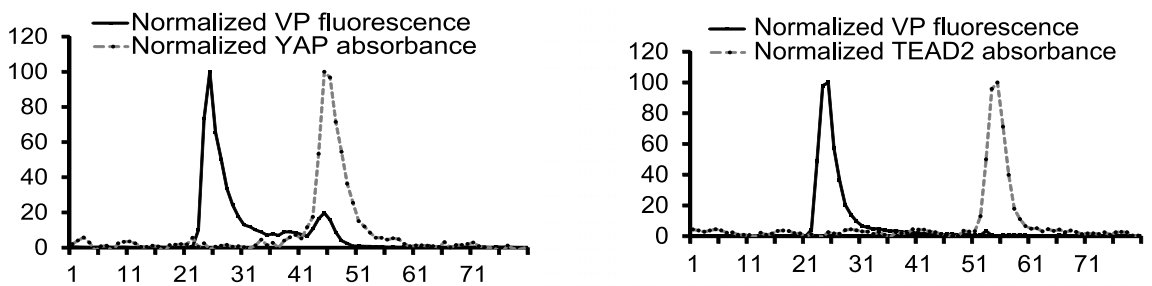

D
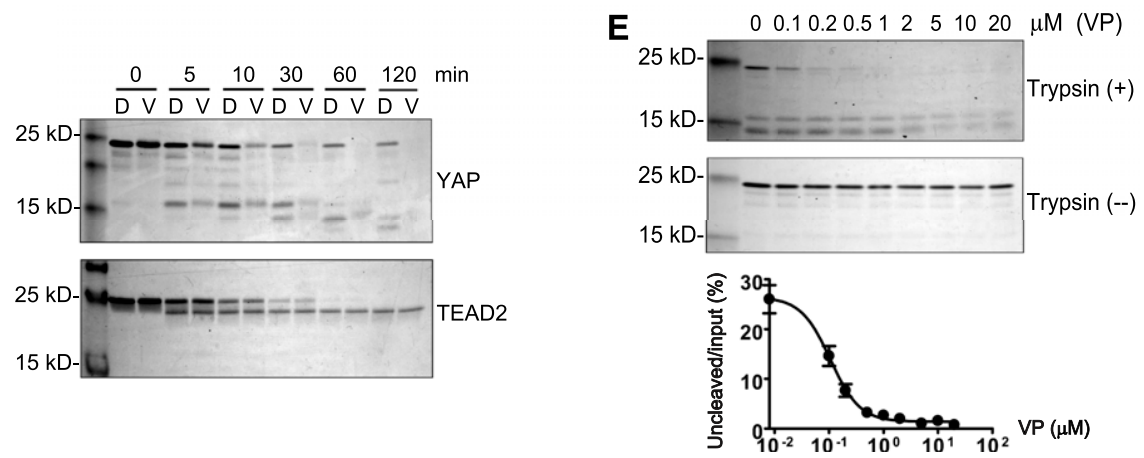

Figure 3. Identification of VP and related porphyrin compounds as inhibitors of TEAD-YAP interactions. $(A)$ Plot showing distribution of luciferase activity of HEK293 cells expressing Gal4-TEAD4/YAP/UAS-Luc and treated with individual Hopkins Library compounds at $10 \mu \mathrm{M}$. All three porphyrin derivatives from the library (PPIX, HP, and VP) were scored as hit compounds as circled. A schematic drawing of the luciferase assay is also shown. $(B)$ Inhibition of TEAD-YAP interaction by VP and PPIX. HEK293 cells expressing Gal4-TEAD4 and HA-YAP were incubated with the indicated concentrations of each chemical, and the presence of Gal4-TEAD in the HA-YAP immunoprecipitates was probed. The chemical structures of VP and PPIX are also shown. $(C)$ VP binds to purified YAP in vitro. Purified YAP (5 $\mu M)$ (left) or TEAD2 (5 $\mu M)$ (right) was incubated with VP $(15 \mu \mathrm{M})$ for $15 \mathrm{~min}$ at room temperature. The mixture was then fractioned through a size exclusion column, and the VP fluorescence was measured in each fraction. Note that an appreciable VP fluorescence coeluted with the YAP protein peak (fraction 45, left) but not with the TEAD2 protein peak (fraction 54, right). (D) Effect of VP on trypsin cleavage of YAP or TEAD2. Purified YAP or TEAD2 $(5 \mu \mathrm{M})$ was preincubated with DMSO (D) or $20 \mu \mathrm{M}$ VP $(\mathrm{V})$ for $30 \mathrm{~min}$ at room temperature prior to trypsin digestion for the indicated time. The cleavage products were analyzed by Coomasie brilliant blue staining after SDS-PAGE. $(E)$ Effect of different concentrations of VP on trypsin cleavage of YAP. YAP $(5 \mu \mathrm{M})$ was preincubated with various concentrations of VP for $30 \mathrm{~min}$ at room temperature, followed by incubation with $(+)$ or without (-) trypsin $(0.1 \mu \mathrm{g} / \mathrm{mL})$ for $1 \mathrm{~h}$ on ice. The graph shows quantification of uncleaved versus input YAP at various VP concentrations.

formed coimmunoprecipitation (co-IP) assays in HEK293 cells expressing GAL4-TEAD4 and HA-YAP that had been treated with the test compounds at various concentrations or the solvent control DMSO (Fig. 3B). While treatment with HP did not cause appreciable inhibition at $10 \mu \mathrm{M}$ (data not shown), treating cells with the other two porphyrins (VP and PPIX) at $10 \mu \mathrm{M}$ dramatically reduced the amount of GAL4-TEAD recovered in the immunoprecipitates of HA-YAP, indicating that these two drugs significantly disrupted YAP-TEAD interactions (Fig. 3B). Interestingly, VP displayed stronger inhibitory effects than PPIX, as $>50 \%$ inhibition could still be observed when VP was titrated down to $2.5 \mu \mathrm{M}$, while 5 $\mu M$ PPIX had negligible effects. Thus, we identified VP and
PPIX as the first small molecule inhibitors targeting the physical interactions between YAP and TEAD.

$\mathrm{VP}$ (trade name Visudyne by Novartis) is used clinically as a photosensitizer in photodynamic therapy for neovascular macular degeneration, where it is activated by a special wavelength laser light to generate reactive oxygen radicals that eliminate the abnormal blood vessels (Michels and Schmidt-Erfurth 2001). The inhibitory activity of VP on YAP-TEAD interactions apparently did not require light activation, since all of our binding assays were conducted in darkness. To investigate how VP inhibits YAP-TEAD interactions, we determined whether VP directly binds to purified YAP or TEAD protein, taking advantage of porphyrin's intrinsic fluorescence 
emission (Rotomskis et al. 1989). Bacterially purified YAP or TEAD2 was incubated with VP, and the mixture was then analyzed on a size exclusion column to examine whether VP coelutes with either protein. An appreciable VP fluorescence coeluted with the YAP protein peak but not with the TEAD2 protein peak, demonstrating that VP selectively binds YAP (Fig. 3C).

We further investigated the binding of VP to YAP or TEAD2 by analyzing proteolytic profiles of the respective protein in the presence of VP, a method that is widely used to determine binding of a small molecule to its target protein and to probe conformational changes of proteins (Yun et al. 2004). Direct binding of a small molecule to a protein can cause conformational changes that are accompanied by alteration in proteolysis patterns of the protein by proteases such as trypsin. Under the optimal cleavage conditions for each protein, $20 \mu \mathrm{M}$ VP significantly enhanced the trypsin cleavage of YAP over time, while it had no effect on the cleavage of TEAD2 (Fig. 3D). In addition, VP dose-dependently accelerated trypsin cleavage of YAP with an $\mathrm{EC}_{50}$ (half maximal effective concentration) value of $0.1 \mu \mathrm{M}$ (Fig. 3E). These results suggest that VP binds to YAP and enhances the accessibility of trypsin to YAP, presumably by changing the conformation of YAP. Taken together, we conclude that VP binds to YAP and changes its conformation, thereby abrogating its interaction with TEAD2.

Given the considerable functional conservation between the mammalian TEAD-YAP complex and its Drosophila counterpart, we tested whether the porphyrins could inhibit the Yki-Sd complex in Drosophila cells. $\mathrm{S}_{2} \mathrm{R}^{+}$cells expressing Myc-Yki and HA-Sd were treated with VP or PPIX, and the amount of HA-Sd recovered in the Myc-Yki immunoprecipitates was measured as an indication of Sd-Yki association. Consistent with their relative potencies against the TEAD-YAP complex in mammalian cells, VP exhibited robust dose-dependent inhibition of Sd-Yki interaction that became apparent at $200 \mathrm{nM}$, whereas PPIX showed a milder inhibitory effect than VP, with an estimated $\mathrm{IC}_{50}$ (half maximal inhibitory concentration) between 5 and $10 \mu \mathrm{M}$ (Supplemental Fig. S7A). VP also inhibited the interaction between endogenous Yki and transiently expressed HA-Sd in S2 cells (Supplemental Fig. S7B). Interestingly, we noted that VP treatment of $S 2$ cells led to a reduction in protein and mRNA levels of endogenous diap1, a direct transcription target of Yki (Supplemental Fig. S7B). These results demonstrate that VP induces transcriptional changes in Drosophila cells that are consistent with inhibition of the Sd-Yki complex.

Next, we examined the ability of VP to inhibit the oncogenic activity of YAP in vivo using two independent assays. In the first assay, we tested VP's ability to suppress YAP-induced hepatomegaly using the inducible YAP transgenic mouse model described above. The ApoErtTA/TRE-YAP mice were injected with VP (100 mg/kg) every other day over a period of $8 \mathrm{~d}$, while these mice were kept on Dox throughout the course of experiment (Fig. 4A). Compared with the control group (Dox+DMSO), the VPtreated group (Dox $+\mathrm{VP})$ exhibited a smaller increase in liver size and a decreased number of mitotic cells (Fig. 4B,C). Importantly, VP treatment did not interfere with YAP transgene expression (Supplemental Fig. S8A) or affect liver size in wild-type control (i.e., nontransgenic) mice (Supplemental Fig. S8B). In the second assay, we tested VP in mice bearing liver-specific knockout of NF2/ Merlin, which exhibited bile duct overproliferation due to activation of endogenous YAP (Zhang et al. 2010). Preg-
A

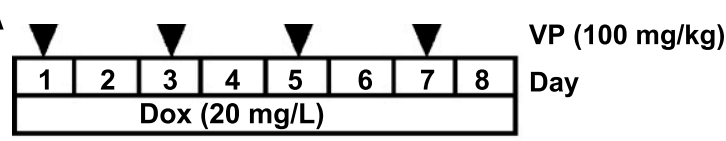

B
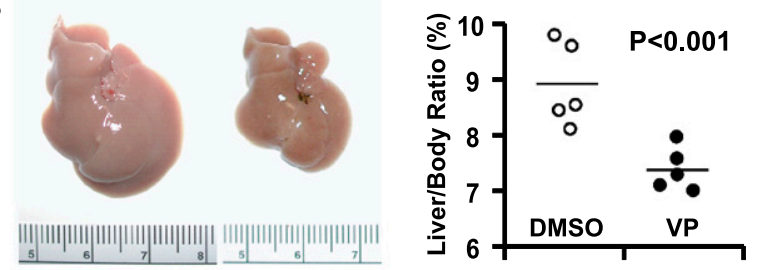

E
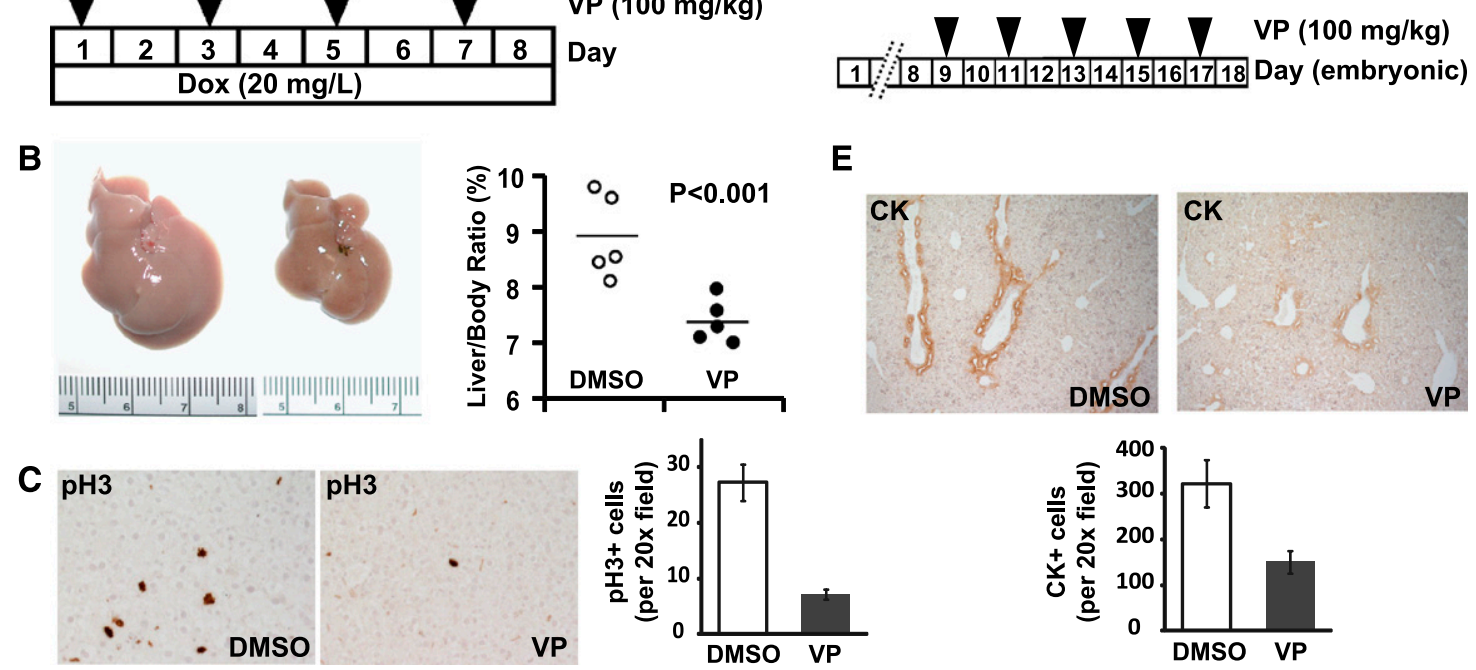

Figure 4. VP suppressed liver overgrowth caused by YAP overexpression or inactivation of Nf2. (A) Experimental design. Three-week-old YAP transgenic mice were fed $20 \mathrm{mg} / \mathrm{L}$ Dox for $8 \mathrm{~d}$. VP suspension $(100 \mathrm{mg} / \mathrm{kg}$ ) was administered by intraperitoneal injection once every other day during the 8-d period. $(B)$ Livers from control (Dox+DMSO; left picture) and VP-treated (Dox+VP; right picture) YAP transgenic mice (left) and quantification of liver-to-body weight ratio (right) at the end of the 8-d period. The VP-treated mice had significantly smaller livers than control livers $(7.3 \%$ vs. $8.9 \%, n=5$ in each group, $P<0.001, t$-test). $(C)$ Phospho-H3 (PH3) staining of liver sections (left) and quantification of PH3-positive cells (right) of liver sections from $B$. (D) Experimental design. Timed pregnant $N f 2^{f l o x 2 / f l o x 2}$ mothers (after mating with $A l b-C r e ; N f 2^{f l o x 2 / f l o x 2}$ males) received intraperitoneal injection of VP $(100 \mathrm{mg} / \mathrm{kg}$ ) or DMSO every other day starting at $9 \mathrm{~d}$ after detection of vaginal plugs (corresponding to E9.5 for embryos carried by the pregnant mothers). Newborn pups (corresponding to E18.5) were analyzed for liver phenotypes. (E) CK staining of liver sections from E18.5 Alb-Cre; Nf2 flox2/flox2 pups that had been subjected to control (DMSO) (left) or VP (right) treatment from E9.5 to E18.5. The graph shows quantification of CK-positive BECs. 
nant mothers bearing Alb-Cre; Nf2 flox2/flox2 embryos received VP injections $(100 \mathrm{mg} / \mathrm{kg})$ every other day starting at embryonic day 9 (E9) of the Alb-Cre; Nf2 flox2/flox2 embryos until birth (Fig. 4D), and BECs were visualized by CK staining at E18.5. As shown in Figure 4E, the number of CK-positive BECs was significantly reduced in VP-treated Nf2-deficent livers compared with control-treated Nf2-deficient livers. Thus, VP suppressed liver overgrowth resulting from either YAP overexpression or activation of endogenous YAP. These results are encouraging, since VP is a clinically applied drug with little side effects, and our results were obtained using an aqueous preparation in which VP bioavailability is suboptimal compared with the lipid-based formulation used in Visudyne.

A major challenge in cancer research is the identification of cellular targets whose inhibitions selectively impair the growth of cancer cells while sparing normal cells (Luo et al. 2009). We demonstrate here that one can exploit the intrinsic property of a growth regulatory pathway; namely, the preferential requirement of TEAD/TEF transcription factors for oncogenic over normal growth as a selective means to inhibit YAP-induced tumorigenesis. The inconsequentiality of TEAD2-DN on normal liver homeostasis suggests that this strategy may offer an excellent "therapeutic window" with minimal adverse effects. As a proof of principle for this approach, we conducted a pilot screen and identified VP as a small molecule that inhibits TEAD-YAP interactions and prevents YAP-induced oncogenic growth. Whether VP or its derivatives are effective on pre-established tumors awaits future investigation. Finally, the efficacy of TEAD2-DN and VP in suppressing Nf2-deficient phenotypes lends further support for a functional link between NF2/Merlin and Hippo signaling (Hamaratoglu et al. 2006; Zhang et al. 2010) and offers the promise of YAP inhibitors as molecular targeted therapeutics for NF2, a genetic disorder caused by inactivation of the NF2/Merlin tumor suppressor.

\section{Materials and methods}

\section{Chemical screen}

Twenty-five-thousand HEK293 cells per well in 96-well plates were transfected (Effectene, Qiagen) with 8 ng of pHA-YAP, pGal4-TEAD4, and $5 \times$ UAS-Luc and $0.4 \mathrm{ng}$ of Pol III renilla luciferase. Library compounds were dispensed into each well immediately after transfection at a final concentration of $10 \mu \mathrm{M}$. After $16 \mathrm{~h}$, cell lysates were examined for firefly luciferase activity relative to renilla luciferase with the Dual-Luciferase Reporter Assay kit (Promega) and a FLUOstar OPTIMA microplate reader (BMG).

\section{Acknowledgments}

This study was supported in part by grants from the NIH (R01EY015708 to D.P., R01AR060636 to S.-J.L., R01DK081417 to R.A.A., and R01 CA122814 to J.O.L.) and FARMRI (to J.O.L). D.P. is an investigator of the Howard Hughes Medical Institute.

\section{References}

Camargo FD, Gokhale S, Johnnidis JB, Fu D, Bell GW, Jaenisch R, Brummelkamp TR. 2007. YAP1 increases organ size and expands undifferentiated progenitor cells. Curr Biol 17: 2054-2060.

Chen L, Chan SW, Zhang X, Walsh M, Lim CJ, Hong W, Song H. 2010. Structural basis of YAP recognition by TEAD4 in the hippo pathway. Genes Dev 24: 290-300.
Chong CR, Xu J, Lu J, Bhat S, Sullivan DJ Jr, Liu JO. 2007. Inhibition of angiogenesis by the antifungal drug itraconazole. ACS Chem Biol 2: 263-270.

Chow L, Berube J, Fromont A, Bell JB. 2004. Ability of scalloped deletion constructs to rescue sd mutant wing phenotypes in Drosophila melanogaster. Genome 47: 849-859.

Dong J, Feldmann G, Huang J, Wu S, Zhang N, Comerford SA, Gayyed MF, Anders RA, Maitra A, Pan D. 2007. Elucidation of a universal sizecontrol mechanism in Drosophila and mammals. Cell 130: 1120-1133.

Halder G, Johnson RL. 2011. Hippo signaling: Growth control and beyond. Development 138: 9-22.

Hamaratoglu F, Willecke M, Kango-Singh M, Nolo R, Hyun E, Tao C, Jafar-Nejad H, Halder G. 2006. The tumour-suppressor genes NF2/ Merlin and Expanded act through Hippo signalling to regulate cell proliferation and apoptosis. Nat Cell Biol 8: 27-36.

Huang J, Wu S, Barrera J, Matthews K, Pan D. 2005. The Hippo signaling pathway coordinately regulates cell proliferation and apoptosis by inactivating Yorkie, the Drosophila homolog of YAP. Cell 122: 421-434.

Lee KP, Lee JH, Kim TS, Kim TH, Park HD, Byun JS, Kim MC, Jeong WI, Calvisi DF, Kim JM, et al. 2010. The Hippo-Salvador pathway restrains hepatic oval cell proliferation, liver size, and liver tumorigenesis. Proc Natl Acad Sci 107: 8248-8253.

Li Z, Zhao B, Wang P, Chen F, Dong Z, Yang H, Guan KL, Xu Y. 2010. Structural insights into the YAP and TEAD complex. Genes Dev 24: 235-240.

Lu L, Li Y, Kim SM, Bossuyt W, Liu P, Qiu Q, Wang Y, Halder G, Finegold MJ, Lee JS, et al. 2010. Hippo signaling is a potent in vivo growth and tumor suppressor pathway in the mammalian liver. Proc Natl Acad Sci 107: 1437-1442.

Luo J, Solimini NL, Elledge SJ. 2009. Principles of cancer therapy: Oncogene and non-oncogene addiction. Cell 136: 823-837.

Michels S, Schmidt-Erfurth U. 2001. Photodynamic therapy with verteporfin: A new treatment in ophthalmology. Semin Ophthalmol 16: 201-206.

Pan D. 2010. The hippo signaling pathway in development and cancer. Dev Cell 19: 491-505.

Rotomskis R, van de Meent EJ, Aartsma TJ, Hoff AJ. 1989. Fluorescence spectra of hematoporphyrin and hematoporphyrin-diacetate aggregates in buffer solution. I Photochem Photobiol B 3: 369-377.

Song H, Mak KK, Topol L, Yun K, Hu J, Garrett L, Chen Y, Park O, Chang J, Simpson RM, et al. 2010. Mammalian Mst1 and Mst2 kinases play essential roles in organ size control and tumor suppression. Proc Nat1 Acad Sci 107: 1431-1436.

Vassilev A, Kaneko KJ, Shu H, Zhao Y, DePamphilis ML. 2001. TEAD/ TEF transcription factors utilize the activation domain of YAP65, a Src/Yes-associated protein localized in the cytoplasm. Genes DeV 15: 1229-1241.

Wu S, Liu Y, Zheng Y, Dong J, Pan D. 2008. The TEAD/TEF family protein Scalloped mediates transcriptional output of the Hippo growth-regulatory pathway. Dev Cell 14: 388-398.

Yun BG, Huang W, Leach N, Hartson SD, Matts RL. 2004. Novobiocin induces a distinct conformation of Hsp90 and alters Hsp90-cochaperone-client interactions. Biochemistry 43: 8217-8229.

Zeng Q, Hong W. 2008. The emerging role of the hippo pathway in cell contact inhibition, organ size control, and cancer development in mammals. Cancer Cell 13: 188-192.

Zhang N, Bai H, David KK, Dong J, Zheng Y, Cai J, Giovannini M, Liu P, Anders RA, Pan D. 2010. The Merlin/NF2 tumor suppressor functions through the YAP oncoprotein to regulate tissue homeostasis in mammals. Dev Cell 19: 27-38.

Zhao B, Ye X, Yu J, Li L, Li W, Li S, Yu J, Lin JD, Wang CY, Chinnaiyan AM, et al. 2008. TEAD mediates YAP-dependent gene induction and growth control. Genes Dev 22: 1962-1971.

Zhao B, Li L, Lei Q, Guan KL. 2010. The Hippo-YAP pathway in organ size control and tumorigenesis: An updated version. Genes Dev 24: 862-874.

Zhou D, Conrad C, Xia F, Park JS, Payer B, Yin Y, Lauwers GY, Thasler W, Lee JT, Avruch J, et al. 2009. Mst1 and Mst2 maintain hepatocyte quiescence and suppress hepatocellular carcinoma development through inactivation of the Yap1 oncogene. Cancer Cell 16: 425-438. 


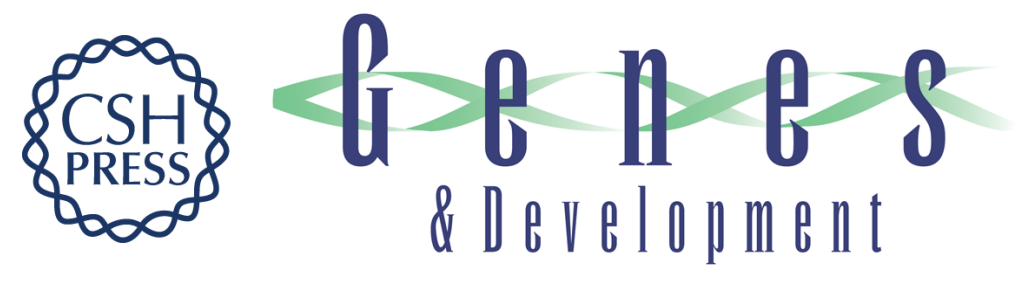

\title{
Genetic and pharmacological disruption of the TEAD-YAP complex suppresses the oncogenic activity of YAP
}

\author{
Yi Liu-Chittenden, Bo Huang, Joong Sup Shim, et al.
}

Genes Dev. 2012, 26: originally published online June 7, 2012

Access the most recent version at doi:10.1101/gad.192856.112

\section{Supplemental http://genesdev.cshlp.org/content/suppl/2012/05/31/gad.192856.112.DC1 \\ Material}

Related Content

References

\section{License}

Email Alerting

Service
This article cites 24 articles, 9 of which can be accessed free at:

http://genesdev.cshlp.org/content/26/12/1300.full.html\#ref-list-1

Articles cited in:

http://genesdev.cshlp.org/content/26/12/1300.full.html\#related-urls

Quit your YAPing: a new target for cancer therapy

Ben Z. Stanger

Genes Dev. June , 2012 26: 1263-1267

Receive free email alerts when new articles cite this article - sign up in the box at the top right corner of the article or click here.

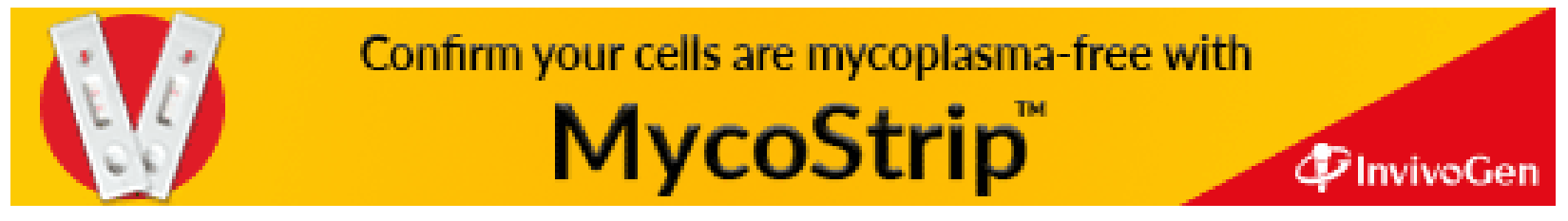

